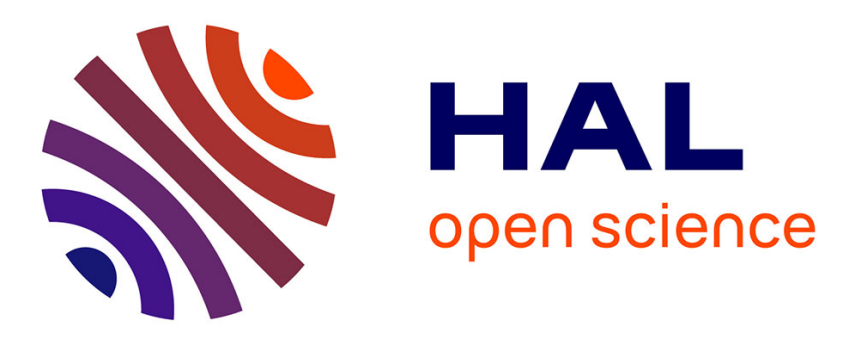

\title{
A multi-arc approach for chaotic orbit determination problems
}

Daniele Serra, Federica Spoto, Andrea Milani

\section{To cite this version:}

Daniele Serra, Federica Spoto, Andrea Milani. A multi-arc approach for chaotic orbit determination problems. Celestial Mechanics and Dynamical Astronomy, 2018, Innovative methods for space threats: from their dynamics to interplanetary missions, 130 (11), pp.75. 10.1007/s10569-018-9868-y . hal01994516

\section{HAL Id: hal-01994516 https://hal.sorbonne-universite.fr/hal-01994516}

Submitted on 25 Jan 2019

HAL is a multi-disciplinary open access archive for the deposit and dissemination of scientific research documents, whether they are published or not. The documents may come from teaching and research institutions in France or abroad, or from public or private research centers.
L'archive ouverte pluridisciplinaire $\mathbf{H A L}$, est destinée au dépôt et à la diffusion de documents scientifiques de niveau recherche, publiés ou non, émanant des établissements d'enseignement et de recherche français ou étrangers, des laboratoires publics ou privés. 


\title{
A multi-arc approach for chaotic orbit determination problems
}

\author{
Daniele Serra $^{\mathrm{a}, *}$, Federica Spoto $^{\mathrm{b}, \mathrm{c}}$, Andrea Milani $^{\mathrm{a}}$ \\ ${ }^{a}$ Dipartimento di Matematica, Università di Pisa, Largo B. Pontecorvo 5, 56127 Pisa, Italy \\ ${ }^{b}$ Université Côte d'Azur, Observatoire de la Côte d'Azur, CNRS, Laboratoire Lagrange, \\ Boulevard de l'Observatoire 06304 Nice, France \\ ${ }^{c} I M C C E$, Observatoire de Paris, PSL Research University, CNRS, Sorbonne Université, \\ UPMC Univ. Paris 06, Univ. Lille 77 av. Denfert-Rochereau 75014, Paris, France
}

\begin{abstract}
Chaotic dynamical systems are characterized by the existence of a predictability horizon, connected to the notion of Lyapunov time, beyond which predictions of the state of the system are meaningless. In order to study the main features of orbit determination in presence of chaos, Spoto and Milani (2016) applied the classical least squares fit and differential correction algorithm to determine a chaotic orbit and a dynamical parameter of a simple discrete system - Chirikov standard map (cf. Chirikov (1979)) - with observations distributed beyond the predictability horizon. They found a time limit beyond which numerical calculations are affected by numerical instability: the computability horizon. In this article we aim at pushing forward such inherent obstacle to numerical calculations in chaotic orbit determination by applying the classical and the constrained multi-arc method (cf. Alessi et al. (2012)) to the same dynamical system. These strategies entail the determination of an orbit when observations are grouped in separate observed arcs. For each arc a set of initial conditions is determined and, in the case of the constrained multi-arc method, all subsequent arcs are constrained to belong to the same trajectory. We show that the use of these techniques in place of the standard least squares method has significant advantages: not only can we perform accurate numerical calculations well beyond the computability horizon, in particular the constrained multi-arc strategy improves considerably the determination of the dynamical parameter.
\end{abstract}

Keywords: chaos, orbit determination, multi-arc strategies, computability horizon, chirikov standard map

*corresponding author

Email address: daniele.serra@dm.unipi.it (Daniele Serra) 


\section{Introduction}

The classical techniques of orbit determination were introduced between the XVIII and the XIX century for the solution of practical problems of celestial mechanics, like the determination of a future position of a newly-discovered small celestial body so as to be able to observe it again. Nowadays, orbit determination methods are also essential for the Radio Science experiments of space missions: from measurements of the range and range-rate of a spacecraft orbiting a planet we can infer the trajectory of the orbiter as well as the value of some parameters characterizing the planet, e.g. the gravity field coefficients, the tidal parameters, the inclination of its rotation axis, etc. In abstract terms, orbit determination can be applied to reconstruct an orbit of a dynamical system, starting from a set of observations. The analytical expression of the dynamical system is supposed to be known, therefore in order to reconstruct the orbit it is sufficient to determine the initial state at an initial time instant, the so-called initial conditions. If the system depends on some other free parameters, the dynamical parameters, they can be recovered with orbit determination as well.

Many of the space-related applications of orbit determination are characterized by chaotic phenomena. For example, chaos appears when an asteroid has a deep close encounter with a planet (in the case of Near Earth Asteroids, especially the Earth) and its main effect is to change the orbit of the object and to increase the uncertainty of its state. Space missions grand tours of planetary systems represent another critical example of arising chaos. As in the case of the NASA/ESA/ASI Cassini-Huygens mission, the many close encounters of the orbiter with Saturn and its moons produced great uncertainty in the determination of the entire orbit, possibly affecting also the determination of some parameters regarding the planet.

Generally speaking, chaotic dynamical systems are characterized by a predictability horizon (cf. Lighthill et al. (1986)), a time limit beyond which it is impossible to forecast the state of the system within a desired accuracy. In the case of orbit determination of a chaotic system, this means that some parameters characterizing the system cannot be determined.

In an attempt of investigating the problem of the determination of chaotic orbits from a mathematically rigorous and abstract point of view, Spoto and Milani (2016) studied a relatively simple model problem in such a way that the results are representative of the real behaviour of more complicated systems, like the ones mentioned above. They considered the dynamical system known as Chirikov standard map (cf. Chirikov (1979)), a discrete system of the two-dimensional torus which has both chaotic and ordered orbits ${ }^{1}$. They simulated a full-cycle orbit de-

\footnotetext{
${ }^{1}$ Since its introduction, Chirikov standard map has been used in Celestial Mechanics as a test problem to understand features of more complicated nearly-integrable and dissipative models
} 
termination process: the observations were iterations of Chirikov standard map plus a Gaussian random error and used the non-linear least squares method, described in Sect. 2.1, to solve the problem of orbit determination of that dynamical system, determining a set of initial conditions and a dynamical parameter. Their goal was to understand the behaviour of the uncertainty in the determination of these parameters, as a function of the number of observations processed. For both chaotic and ordered orbits, they obtained that the uncertainties of the parameters decrease as a polynomial function of the number of observations. However, in the chaotic case they found that there exists a maximum number of observations that can be processed before the computation becomes numerically unstable. Such time limit is called computability horizon. It strongly depends on the chaoticity of the system, and it is a consequence of the numerical instability of the orbit computation. The computability horizon is therefore a realization of the predictability horizon when dealing with numerical experiments: its value, which depends on the Lyapunov exponent of the orbit, can be computed by a simple empirical formula. No computability horizon was found in the ordered case.

The first goal of this paper is to explore a different numerical technique to overcome the limitation coming from the computability horizon. To be able to compare our outcomes with the previous results, we consider the same problem model studied in Spoto and Milani (2016). The novelty is the introduction of a multi-arc approach in the orbit determination. The idea of the classical pure multi-arc method (Sect. 2.2) is to consider observations grouped in separate and relatively short arcs, and determine initial conditions for each arc. If we also impose the constraint that all arcs belong to the same trajectory, we obtain the constrained multi-arc strategy (introduced by Alessi et al. (2012) for the orbit determination of the ESA/JAXA BepiColombo mission to Mercury). We analyze the case when, in addition to the initial conditions, we also solve for a global dynamical parameter, shared by all arcs. The main advantage of multi-arc methods is that numerical computations are performed arc by arc, therefore we can choose the number of observations of each arc in such a way that the computability horizon is never met. We also aim to study the behaviour of the formal uncertainty associated with the solve-for parameters. We will be particularly interested in assessing whether the constrained multi-arc method provides an improvement in the determination of the dynamical parameter with respect to the pure multi-arc method.

This paper is organized as follows. In Sect. 2 we recall classical notions of orbit determination, multi-arc techniques and introduce the notation used throughout

(cf. Celletti et al. (2010)). Moreover, it locally approximates the Kepler map, an area-preserving map describing the motion of a celestial body on a nearly-parabolic orbit (cf. Petrosky (1986), Chirikov and Vecheslavov (1986) and Schevchenko (2011)). 
the article. Sect. 3 describes our framework and presents the mathematical formulation of the pure and constrained multi-arc techniques in the case of Chirikov standard map. In Sect. 4 we set up the numerical experiment, show the results obtained, and discuss the differences between one chaotic example and one belonging to an ordered region. Finally, in Sect. 5 we draw our conclusions and hint at possible applications of this work.

\section{Orbit determination}

The scope of this section is to provide the essential notions of orbit determination, non-linear least-squares and multi-arc strategies, as well as to introduce the terminology used in the rest of the article. The reader may refer to Milani and Gronchi (2010) for a comprehensive discussion on the subject.

\subsection{Non-linear least squares}

Orbit determination indicates the process of reconstructing the orbit of a dynamical system (possibly dependent on some dynamical parameters $\boldsymbol{\mu}$ )

$$
\left\{\begin{array}{l}
\dot{\mathbf{x}}=\mathbf{f}(t, \mathbf{x}, \boldsymbol{\mu}) \\
\dot{\mathbf{x}}\left(t_{0}\right)=\mathbf{x}_{0}
\end{array}\right.
$$

starting from a set of observations $r_{1}, \ldots, r_{m}$ at times $t_{1}, \ldots, t_{m}$. Since the functional expression $\mathbf{f}$ on the right-hand side is usually known, the orbit $\mathbf{x}(t)$ is uniquely determined by $\mathbf{x}_{0}$ and $\boldsymbol{\mu}$. Observations are not necessarily made up of direct measurements of the state of the system, but can be a function of it. For instance, in the case of a planetary orbiter the goal is to determine the planetocentric position and velocity of the spacecraft, but the observations are most commonly range (radial distance from the Earth) or range-rate (radial velocity w.r.t. the Earth) measurements.

To model the observations we use a prediction function $r\left(t, \mathbf{x}_{0}, \boldsymbol{\mu}\right)$, depending on the time $t$, the unknown initial conditions $\mathbf{x}_{0}$ and the dynamical parameters $\boldsymbol{\mu}$. Note that the distinction between initial conditions and dynamical parameters is purely formal: for this reason, in the following we will use the term "parameters" to refer to either $\mathbf{x}_{0}$ or $\boldsymbol{\mu}$. The residuals are defined as

$$
\xi_{i}=r_{i}-r\left(t_{i}, \mathbf{x}_{0}, \boldsymbol{\mu}\right), \quad i=1, \ldots, m,
$$

thus the vector of the residuals $\boldsymbol{\xi}=\left(\xi_{i}\right)$ is a function of $\mathbf{x}_{0}$ and $\boldsymbol{\mu}$. The least-squares method allows to compute a solution for a desired subset $\mathbf{u}$ of the parameters (the solve-for parameters), along with some statistical information associated with it. 
The solution of the orbit determination problem should - in some sense - minimize the residuals. More rigorously, let us define the weighted target function

$$
Q(\mathbf{u})=\frac{1}{m} \sum_{i=1}^{m} \frac{\xi_{i}^{2}(\mathbf{u})}{\sigma_{i}^{2}},
$$

where $\sigma_{i}^{-1}$ is the weight ${ }^{2}$ associated to observation $r_{i}$. The least-squares method states that the solution $\mathbf{u}$ is a local minimum point of $Q$. This leads to the differential correction algorithm, that is an iterative procedure to compute $\mathbf{u}$ : we start from an initial guess $\mathbf{u}_{0}$ and generate a sequence $\left(\mathbf{u}_{j}\right)$, where $\mathbf{u}_{j+1}$ is obtained solving the normal equations:

$$
C\left(\mathbf{u}_{j+1}-\mathbf{u}_{j}\right)=D, \quad j \geq 0
$$

where $B=\partial \boldsymbol{\xi} / \partial \mathbf{u}$ is called design matrix, $C=B^{T} W B$ is called normal matrix, $D=-B^{T} W \boldsymbol{\xi}$ and $W=\operatorname{diag}\left(\sigma_{1}^{-2}, \ldots, \sigma_{m}^{-2}\right)$ is the weight matrix. At convergence, the obtained value $\mathbf{u}^{*}$ is the solution of the orbit determination problem. The statistical information about $\mathbf{u}^{*}$ are contained in the covariance matrix $\Gamma=C^{-1}$ : the formal uncertainty of parameter $u_{i}$ is $\sqrt{\Gamma_{i i}}$, and the correlation between two parameters $u_{i}$ and $u_{j}$ is $\Gamma_{i j} /\left(\sqrt{\Gamma_{i i}} \sqrt{\Gamma_{j j}}\right)$.

When some information on one or more solve-for parameters is available, it may be taken into account during the differential corrections process. Not only is this useful to stabilize the convergence of the algorithm, but it is also the only way to cure a possible rank deficiency of that specific orbit determination problem. Let $\mathbf{u}_{P}$ be the apriori value of the $N$ parameters that we want to include in the fit. With each apriori observation $u_{i}=u_{P, i}, i=1, \ldots, N$ is associated an apriori standard deviation $\sigma_{P, i}, i=1, \ldots, N$. If $C_{P}=\operatorname{diag}\left(\sigma_{P, 1}^{-2}, \ldots, \sigma_{P, N}^{-2}\right)$, adding apriori observations $\mathbf{u}=\mathbf{u}_{P}$ can be realized modifying the target function $Q$ as follows:

$$
Q(\mathbf{u})=\frac{1}{m+N}\left[\boldsymbol{\xi}^{T} W \boldsymbol{\xi}+\left(\mathbf{u}-\mathbf{u}_{P}\right)^{T} C_{P}\left(\mathbf{u}-\mathbf{u}_{P}\right)\right] .
$$

As a consequence, the normal equations considering the apriori observations are:

$$
\left(C+C_{P}\right)\left(\mathbf{u}_{j+1}-\mathbf{u}_{j}\right)=D+C_{P}\left(\mathbf{u}_{j}-\mathbf{u}_{P}\right) .
$$

\subsection{Multi-arc strategies}

In the case of planetary space missions with an orbiter, the spacecraft is never continuously tracked from Earth due to visibility conditions, mission design, body

\footnotetext{
${ }^{2}$ Weighing observations is a common technique used to take into account the possibility that some observations are more accurate than the others, for instance because they were realized with better instrumentation.
} 
occultations of the probe, etc., therefore observations are naturally divided into arcs, separated by intervals in the dark. Dark periods are extended in time (days or even weeks) and scheduled manoeuvres often occur in that time. The uncertainty introduced by such manoeuvres and the difficulty of modeling the long-term effects of non-gravitational perturbations make the orbit determination process a challenging task. One possible solution is to use a classical (in the following, pure) multi-arc method: we can neglect all dynamics happening in the dark periods as long as we determine - in addition to the dynamical parameters - a set of initial conditions of the probe for each observed $\mathrm{arc}^{3}$. For the practical calculation of the prediction function, this means that numerical propagation of the state of the spacecraft is made arc by arc. On the contrary, in a single-arc strategy the initial conditions have to be propagated for the entire duration of the mission.

Let us shortly describe the mathematical formulation of the pure multi-arc method. Let us suppose that the observations are divided in $n$ disjoint subsets, called arcs, and let $\mathbf{x}_{0}^{k}$ be the initial conditions vector of arc $k, k=1, \ldots, n$. Let us divide the set of parameters in two subsets: the global parameters $\mathbf{g}$, which are shared by all arcs, and the local parameters $\mathbf{h}_{1}, \ldots, \mathbf{h}_{k}$, which are characteristic of the single arcs (e.g. $\mathbf{h}_{k}$ includes $\mathbf{x}_{0}^{k}$ ). Moreover, the local parameters from one arc do not influence the dynamics of the other arcs. The residuals vector $\boldsymbol{\xi}$ is divided in $k$ subvectors:

$$
\boldsymbol{\xi}\left(\mathbf{g}, \mathbf{h}_{1}, \ldots, \mathbf{h}_{k}\right)=\left(\boldsymbol{\xi}^{1}\left(\mathbf{g}, \mathbf{h}_{1}\right), \ldots, \boldsymbol{\xi}^{k}\left(\mathbf{g}, \mathbf{h}_{k}\right), \ldots, \boldsymbol{\xi}^{n}\left(\mathbf{g}, \mathbf{h}_{n}\right)\right)
$$

and consequently

$$
\frac{\partial \boldsymbol{\xi}^{i}}{\partial \mathbf{h}_{j}}=\mathbf{0}, \quad \text { for } i \neq j .
$$

The normal matrix inherits an arrow-like structure:

$$
C=\left[\begin{array}{cccc}
C_{\mathbf{g g}} & C_{\mathbf{g h}_{1}} & \ldots & C_{\mathbf{g h}_{n}} \\
C_{\mathbf{h}_{1} \mathbf{g}} & C_{\mathbf{h}_{1} \mathbf{h}_{1}} & \mathbf{0} & \mathbf{0} \\
\vdots & \mathbf{0} & \ddots & \mathbf{0} \\
C_{\mathbf{h}_{n} \mathbf{g}} & \mathbf{0} & \mathbf{0} & C_{\mathbf{h}_{n} \mathbf{h}_{n}}
\end{array}\right]
$$

where

$$
\begin{gathered}
C_{\mathbf{g g}}=\sum_{k=1}^{n}\left(B_{\mathbf{g}}^{k}\right)^{T} W^{k} B_{\mathbf{g}}^{k} \\
C_{\mathbf{g h}_{k}}=\left(B_{\mathbf{g}}^{k}\right)^{T} W^{k} B_{\mathbf{h}_{k}}^{k}=C_{\mathbf{h}_{k} \mathbf{g}}^{T}, \quad C_{\mathbf{h}_{k} \mathbf{h}_{k}}=\left(B_{\mathbf{h}_{k}}^{k}\right)^{T} W^{k} B_{\mathbf{h}_{k}}^{k}, \quad k=1, \ldots, n,
\end{gathered}
$$

\footnotetext{
${ }^{3}$ From another point of view, this is equivalent to having a different spacecraft for each arc.
} 
$W^{k}$ is the weight matrix of arc $k$, and the design sub-matrices are

$$
B_{\mathbf{g}}^{k}=\frac{\partial \boldsymbol{\xi}^{k}}{\partial \mathbf{g}}, \quad B_{\mathbf{h}_{k}}^{k}=\frac{\partial \boldsymbol{\xi}^{k}}{\partial \mathbf{h}_{k}}, \quad k=1, \ldots, n .
$$

Finally, the right-hand side of the normal equations $D$ is divided into sub-vectors:

$$
\begin{aligned}
D_{\mathbf{g}} & =-\sum_{k=1}^{n}\left(B_{\mathbf{g}}^{k}\right)^{T} W^{k} \boldsymbol{\xi}^{k} \\
D_{\mathbf{h}_{k}} & =-\left(B_{\mathbf{h}_{k}}^{k}\right)^{T} W^{k} \boldsymbol{\xi}^{k}, \quad k=1, \ldots, n .
\end{aligned}
$$

This particular structure can be exploited to split the normal equations (1) in smaller systems, which are more convenient to solve from a numerical point of view.

Alessi et al. (2012) presented a new version of the multi-arc method for the Radio Science Experiment of the ESA/JAXA BepiColombo mission (cf. Benkhoff et al. (2010)), called constrained multi-arc strategy. This is based on the idea that all arcs belong to the same orbit, therefore they should be connected in a smooth way. The connection is established as follows: for every pair of subsequent arcs, their initial conditions are propagated to the central time of the dark interval between them, and the difference between the two resulting states is constrained to be smaller than the observation noise (see later, Fig. 1). In the case of interplanetary missions, such strategy is more difficult to implement since the dynamical model of the spacecraft has to take into account the perturbations occurring in the dark periods. On the other hand, using such approach improves considerably the stability of the entire orbit determination process. The rigorous mathematical formulation of the constrained multi-arc method will be given in the next section for the model problem we are considering.

\section{Multi-arc strategies for Chirikov standard map}

In this section we describe the mathematical formulation of the pure and constrained multi-arc methods for the orbit determination problem of Chirikov standard map.

Let us start recalling the definition of the dynamical system we are dealing with. Chirikov standard map is a conservative discrete dynamical system, defined on a two-dimensional torus by the following formula:

$$
S_{\mu_{0}}^{i+1}\left[\left(x_{0}, y_{0}\right)\right]=\left\{\begin{array}{l}
x_{i+1}=x_{i}+y_{i+1} \\
y_{i+1}=y_{i}-\mu_{0} \sin \left(x_{i}\right), \quad i \in \mathbb{N},
\end{array}\right.
$$


where $S_{\mu_{0}}^{0}\left[\left(x_{0}, y_{0}\right)\right]=:\left(x_{0}, y_{0}\right)$ are the initial conditions and $\mu_{0}$ is a parameter. The system could have both ordered and chaotic orbits, depending on the choice of $\left(x_{0}, y_{0}\right)$ and $\mu_{0}$. There are more regular orbits for smaller values of $\mu_{0}$, whereas larger values of this parameter result in more chaotic orbits.

\subsection{Pure multi-arc method}

The first element we need for setting up an orbit determination process are the observations. Since we are aiming at using a multi-arc method, we need to reproduce the same natural observational framework where multi-arc techniques are used in practice, thus we need observations organized in arcs and dark periods between them. As done in Spoto and Milani (2016), the observations are simply iterations of the dynamical system and are obtained by means of the following procedure. Firstly, we compute forward and backward iterations of (2) starting from the initial conditions $\left(x_{0}, y_{0}\right)$. Secondly, we add a random noise, modeled as a Gaussian distribution $\mathcal{N}(0, \sigma)$, obtaining $\left(\bar{x}_{i}, \bar{y}_{i}\right)=\left(\mathcal{N}\left(x_{i}, \sigma\right), \mathcal{N}\left(y_{i}, \sigma\right)\right)$. From this sequence of modified iterates, we extract observations grouped in $2 n+1$ observed arcs, numbered from $-n$ to $n$, in such a way that:

- each arc $k$ contains $2 m+1$ observations $\left(\bar{x}_{j}, \bar{y}_{j}\right)^{k}, j=-m, \ldots, m$, and $\left(\bar{x}_{0}, \bar{y}_{0}\right)^{k}$ denotes the central point of each arc;

- $\left(\bar{x}_{0}, \bar{y}_{0}\right):=\left(\bar{x}_{0}, \bar{y}_{0}\right)^{0}$ is the central point of arc 0 ;

- any two subsequent arcs are separated by a dark interval composed of $2 m_{d}+1$ iterates, numbered from $-m_{d}$ to $m_{d}$.

Consequently, the total number of observations is $(2 n+1)(2 m+1)$. Since the observations are practically iterations of the map with a noise, in the following the word "iteration" will be used as a synonym of "observation".

The prediction function is given by the standard map itself, depending on the dynamical parameter $\mu_{0}$ and the central point of each arc $\left(x_{0}, y_{0}\right)^{k}$, which we will consider from now on initial conditions of the arc. The set of the solve-for parameters in the orbit determination process is $\left\{\mu_{0},\left(x_{0}, y_{0}\right)^{k}, k=-n, \ldots, n\right\}$. As already stated before, the parameters can be divided into global and local parameters. In our case, the vector of the global parameters $\mathbf{g}$ is only the dynamical parameter $\mu_{0}$, whereas the vector of the local parameters $\mathbf{h}$ is the vector of all the initial conditions $\left\{\left(x_{0}, y_{0}\right)^{k}, k=-n, \ldots, n\right\}$, which can be split in subvectors $\mathbf{h}_{k}=\left(x_{0}, y_{0}\right)^{k}, k=-n, \ldots, n$.

The core of the least squares fit is the minimization of the target function

$$
Q=\frac{1}{(2 n+1)(2 m+1)} \sum_{k=-n}^{n}\left(\boldsymbol{\xi}^{k}\right)^{T} W^{k} \boldsymbol{\xi}^{k}
$$




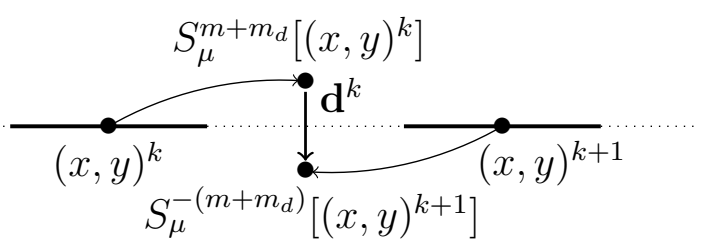

Figure 1: The jump vector $\mathbf{d}^{k}$ between arcs $k$ and $k+1$ given by (5) is the difference between the backward-propagated current initial conditions of arc $k+1$, namely $(x, y)^{k+1}$, and the forwardpropagated current initial conditions of arc $k$, namely $(x, y)^{k}$, to the central point of the dark interval between the two arcs.

where $W^{k}$ is the weight matrix of arc $k$ and $\boldsymbol{\xi}^{k}=\left(\boldsymbol{\xi}_{-m}^{k}, \ldots, \boldsymbol{\xi}_{m}^{k}\right)$ are the residuals relative to arc $k$, which read

$$
\boldsymbol{\xi}_{j}^{k}=\left(\bar{x}_{j}, \bar{y}_{j}\right)^{k}-S_{\mu}^{j}\left[(x, y)^{k}\right], \quad j=-m, \ldots, m .
$$

Here $(x, y)^{k}=\left(x_{0}+\delta x, y_{0}+\delta y\right)^{k}$ and $\mu=\mu_{0}+\delta \mu$ are the current guess of the parameters. The crucial property characterizing the multi-arc strategies is described by

$$
\frac{\partial \boldsymbol{\xi}^{k_{1}}}{\partial\left(x_{0}, y_{0}\right)^{k_{2}}}=\mathbf{0}, \quad \text { for all } k_{1} \neq k_{2}, k_{1}, k_{2}=-n, \ldots, n,
$$

which expresses the fact that the residuals from one arc do not depend on the local parameters of another arc. We do not report the expressions of the normal equations and the matrices involved as they are formally identical to those in Sect. 2.2, except for summations, now running over $k=-n, \ldots, n$. Note that these matrices have explicit formulae and they can be computed as shown in Spoto and Milani (2016), Sect. 2.

\subsection{Constrained multi-arc}

Let us now show the mathematical formulation of the constrained multi-arc method for Chirikov standard map. The observations, the prediction function and the residuals are the same as in the classical multi-arc strategy. The main idea is to impose the constraint that two subsequent arcs belong to the same orbit.

We define the jump vector between arcs $k$ and $k+1$ as (see Fig. 1)

$$
\mathbf{d}^{k}:=S_{\mu}^{-\left(m+m_{d}\right)}\left[(x, y)^{k+1}\right]-S_{\mu}^{m+m_{d}}\left[(x, y)^{k}\right], \quad k=-n, \ldots, n-1
$$

where $S$ is given by $(2),(x, y)^{k}$ and $(x, y)^{k+1}$ are the current guess of the initial conditions of arc $k$ and $k+1$ respectively, and $\mu$ is the current guess of the dynamical parameter. Constraining two consecutive arcs to belong to the same orbit 
is equivalent to including the following apriori observations to the fit:

$$
\mathbf{d}^{k}=\mathbf{0}
$$

with associated apriori standard deviation $\sigma_{P}$. This corresponds to adding $2 n$ quadratic forms to the target function (3), which now reads

$$
\widetilde{Q}=\frac{1}{(2 n+1)(2 m+1)+4 n}\left(\sum_{k=-n}^{n}\left(\boldsymbol{\xi}^{k}\right)^{T} W^{k} \boldsymbol{\xi}^{k}+\frac{1}{\sigma_{P}^{2}} \sum_{k=-n}^{n-1}\left(\mathbf{d}^{k}\right)^{T} \mathbf{d}^{k}\right)
$$

The constrained multi-arc design matrix contains also the derivative of the jump vectors with respect to the parameters:

$$
\begin{aligned}
\frac{\partial \mathbf{d}^{k}}{\partial \mathbf{g}} & = \begin{cases}\frac{\partial S_{\mu}^{-\left(m+m_{d}\right)}\left[(x, y)^{k+1}\right]}{\partial \mu_{0}}-\frac{\partial S_{\mu}^{m+m_{d}}\left[(x, y)^{k}\right]}{\partial \mu_{0}} & k=-n, \ldots, n-1 \\
\mathbf{0} & k=n\end{cases} \\
\frac{\partial \mathbf{d}^{k-1}}{\partial \mathbf{h}_{k}} & = \begin{cases}\frac{\partial S_{\mu}^{-\left(m+m_{d}\right)}\left[(x, y)^{k}\right]}{\partial\left(x_{0}, y_{0}\right)^{k}} & k=-n+1, \ldots, n \\
\mathbf{0} & k=-n\end{cases} \\
\frac{\partial \mathbf{d}^{k}}{\partial \mathbf{h}_{k}} & = \begin{cases}-\frac{\partial S_{\mu}^{m+m_{d}}\left[(x, y)^{k}\right]}{\partial\left(x_{0}, y_{0}\right)^{k}} & k=-n, \ldots, n-1 \\
\mathbf{0} & k=n .\end{cases}
\end{aligned}
$$

The constrained multi-arc normal matrix $\widetilde{C}$ results in a block tridiagonal arrowshaped matrix:

$$
\widetilde{C}=\left[\begin{array}{ccccc}
\widetilde{C}_{\mathbf{g g}} & \widetilde{C}_{\mathbf{g h}_{-n}} & \ldots & \ldots & \widetilde{C}_{\mathbf{g h}_{n}} \\
\widetilde{C}_{\mathbf{h}_{-n} \mathbf{g}} & \widetilde{C}_{\mathbf{h}_{-n} \mathbf{h}_{-n}} & \widetilde{C}_{\mathbf{h}_{-n} \mathbf{h}_{-n+1}} & \mathbf{0} & \mathbf{0} \\
\vdots & \widetilde{C}_{\mathbf{h}_{-n+1} \mathbf{h}_{-n}} & \ddots & \ddots & \vdots \\
\vdots & \mathbf{0} & \ddots & \ddots & \widetilde{C}_{\mathbf{h}_{n-1} \mathbf{h}_{n}} \\
\widetilde{C}_{\mathbf{h}_{n} \mathbf{g}} & \mathbf{0} & \mathbf{0} & \widetilde{C}_{\mathbf{h}_{n} \mathbf{h}_{n-1}} & \widetilde{C}_{\mathbf{h}_{n} \mathbf{h}_{n}}
\end{array}\right] ;
$$


where

$$
\begin{aligned}
& \widetilde{C}_{\mathbf{g g}}=C_{\mathbf{g g}}+\frac{1}{\sigma_{P}^{2}} \sum_{k=-n}^{n-1}\left(\frac{\partial \mathbf{d}^{k}}{\partial \mathbf{g}}\right)^{T} \frac{\partial \mathbf{d}^{k}}{\partial \mathbf{g}} \\
& \widetilde{C}_{\mathbf{g h}_{k}}=C_{\mathbf{g h}_{k}}+\frac{1}{\sigma_{P}^{2}}\left(\frac{\partial \mathbf{d}^{k}}{\partial \mathbf{g}}\right)^{T} \frac{\partial \mathbf{d}^{k}}{\partial \mathbf{h}_{k}}+\frac{1}{\sigma_{P}^{2}}\left(\frac{\partial \mathbf{d}^{k-1}}{\partial \mathbf{g}}\right)^{T} \frac{\partial \mathbf{d}^{k-1}}{\partial \mathbf{h}_{k}}=\widetilde{C}_{\mathbf{h}_{k} \mathbf{g}}^{T}, \quad k=-n, \ldots, n \\
& \widetilde{C}_{\mathbf{h}_{k} \mathbf{h}_{k}}=C_{\mathbf{h}_{k} \mathbf{h}_{k}}+\frac{1}{\sigma_{P}^{2}}\left(\frac{\partial \mathbf{d}^{k}}{\partial \mathbf{h}_{k}}\right)^{T} \frac{\partial \mathbf{d}^{k}}{\partial \mathbf{h}_{k}}+\frac{1}{\sigma_{P}^{2}}\left(\frac{\partial \mathbf{d}^{k-1}}{\partial \mathbf{h}_{k}}\right)^{T} \frac{\partial \mathbf{d}^{k-1}}{\partial \mathbf{h}_{k}}, \quad k=-n, \ldots, n \\
& \widetilde{C}_{\mathbf{h}_{k-1} \mathbf{h}_{k}}=\frac{1}{\sigma_{P}^{2}}\left(\frac{\partial \mathbf{d}^{k-1}}{\partial \mathbf{h}_{k-1}}\right)^{T} \frac{\partial \mathbf{d}^{k-1}}{\partial \mathbf{h}_{k}}=\widetilde{C}_{\mathbf{h}_{k+1} \mathbf{h}_{k}}, \quad k=-n+1, \ldots, n .
\end{aligned}
$$

Finally, the constrained multi-arc right-hand side is

$$
\begin{aligned}
\widetilde{D}_{\mathbf{g}} & =D_{\mathbf{g}}-\frac{1}{\sigma_{P}^{2}} \sum_{k=-n}^{n-1} \frac{\partial \mathbf{d}^{k}}{\partial \mathbf{g}} \mathbf{d}^{k} \\
\widetilde{D}_{\mathbf{h}_{k}} & =D_{\mathbf{h}_{k}}-\frac{1}{\sigma_{P}^{2}}\left(\frac{\partial \mathbf{d}^{k}}{\partial \mathbf{h}_{k}}\right)^{T} \mathbf{d}^{k}-\frac{1}{\sigma_{P}^{2}}\left(\frac{\partial \mathbf{d}^{k-1}}{\partial \mathbf{h}_{k}}\right)^{T} \mathbf{d}^{k-1}, \quad k=-n, \ldots, n .
\end{aligned}
$$

\section{Numerical experiments}

Spoto and Milani (2016) showed that the determination of a Chirikov standard map's orbit with initial conditions $\left(x_{0}, y_{0}\right)=(3,0)$, using the classical singlearc method and determining $\left(x_{0}, y_{0}\right)$ and the dynamical parameter $\mu_{0}$, is limited by the computability horizon. For that orbit and in double precision, after $\sim$ 200 iterations of the map (i.e, when processing more than $\sim 200$ observations), computation becomes numerically unstable. Since the Lyapunov time ${ }^{4}$ of that orbit is about 11 iterations, the limit in this case corresponds to about 200/11 18 Lyapunov times. Using a multi-arc strategy is a way to overcome this limitation: since such technique entails that the computation of the prediction function (which also requires numerical propagations of the dynamical system) is performed arc by arc, if the number of observations belonging to each arc is small enough, the computability horizon is never reached and numerical instability is thus avoided. In our experiments with the same dynamical system and the same chaotic orbit, we push orbit determination up to more than 60 Lyapunov times in double precision, as described in the following.

\footnotetext{
${ }^{4}$ The Lyapunov time of an orbit is the inverse of the largest Lyapunov exponent of that orbit and indicates the time after which the distance between two nearby trajectories increase by a factor $e$. The computation of the Lyapunov times of the orbits of Chirikov standard map is described in Spoto and Milani (2016).
} 


\subsection{Setup of the experiment}

We create the observations as explained in Sec. 3. We use $\sigma=10^{-8}$ as the Gaussian noise standard deviation, and we choose $m=5, m_{d}=1, n=50$. In this way each arc is composed of 11 observations - approximately 1 Lyapunov time -, the dark intervals are composed of 3 iterations, and the total number of arcs is 101. The number of iterations required for simulating the observables is $\sim 700$, forward and backward, which correspond to more than 60 Lyapunov times of the orbit with initial conditions $(3,0)$. With this choice of $m$ and $m_{d}$ we are able to avoid the computability horizon.

We apply the least squares fit and the differential corrections algorithm via pure and constrained multi-arc approaches. As already described in Sect. 2, the differential corrections algorithm consists of solving iteratively the normal equations until a given convergence criterion is satisfied. We will discuss such criterion in Sec. 4.2. The solve-for parameters are the initial conditions $\left(x_{0}, y_{0}\right)^{k}, k=-n, \ldots, n$ of the arcs and the dynamical parameter $\mu_{0}$.

In order to ensure stability of the process, we tackle orbit determination in a progressive fashion. At step 0 we process only arc 0 , solving for the initial conditions of this arc and $\mu_{0}$. For each parameter we use as first guess its true value (i.e. the value used to create the observables) incremented by $10 \sigma$. We do this to simulate a real orbit determination case, where the first guess of the parameters is usually very different from the value at convergence. Once convergence is reached, we have corrected values for such parameters. We use them in step 1 as new first guess in an orbit determination process in which we now process, along with arc 0 , also arcs 1 and -1 , and we solve for the initial conditions of the three arcs and $\mu_{0}$. The first guess for the initial conditions of the newly added arcs is given by their simulation value plus $10 \sigma$, as in the previous steps. In general, at step $k+1$ we add arcs $-k-1$ and $k+1$ to the set of the previously processed $2 k+1$ arcs (from $-k$ to $k$ ) and solve for the initial conditions of all arcs and $\mu_{0}$. The first guess values of the parameters are chosen as done in the previous steps. We repeat such scheme until the total number of arcs is reached.

\subsection{Convergence conditions}

The differential corrections entail the iterative solution of the normal equations, therefore it is crucial to establish a criterion for the termination of the algorithm. The principle of convergence is based on two conditions: on the one hand, we want to make sure that the last correction of the parameters (i.e. the difference between the value of the parameters at the present iteration and at the previous one) is "small", in a sense we are going to specify; on the other hand, when coping with a constrained multi-arc strategy, we need to ensure that the jump vectors are not larger than the observation noise, say at least ten times smaller. These two specifications translate into the following mathematical conditions: 1) if $\Delta \mathbf{u}$ is the 
last correction to the solve-for parameters, we require that its norm $\|\Delta \mathbf{u}\|_{C}(C$ is the normal matrix of the adopted multi-arc strategy) satisfies

$$
\|\Delta \mathbf{u}\|_{C}:=\sqrt{\frac{\Delta \mathbf{u}^{T} C \Delta \mathbf{u}}{(2 n+1)(2 m+1)}} \ll 1,
$$

meaning that all following iterations will not improve significantly the solution; 2) in order to have a smooth orbit, the norm of the jump vectors should be negligible with respect to the observation noise $\sigma$, resulting in

$$
d_{\mathrm{RMS}}:=\sqrt{\sum_{k=-n}^{n-1} \frac{\left\|\mathbf{d}^{k}\right\|^{2}}{4 n}} \leq \sigma^{*},
$$

where $\left\|\mathbf{d}^{k}\right\|$ is the euclidean norm of $\mathbf{d}^{k}$ and $\sigma^{*}$ is a fraction of $\sigma$. The same value $\sigma^{*}$ is considered for the apriori standard deviation $\sigma_{P}$ associated with the contraints. We adopt a value of $\sigma_{P}$ which varies along with the iteration of the fit:

$$
\sigma_{P}=\max \left(\frac{d_{\mathrm{RMS}}}{100}, \sigma^{*}\right) .
$$

\subsection{Results: chaotic case}

The results presented in this section refer to the determination of an orbit of Chirikov standard map simulated with initial conditions $\left(x_{0}, y_{0}\right)=(3,0)$ and $\mu_{0}=0.5$, with the setup described in Sect. 4.1. Such an orbit belongs to a chaotic region of the phase space of the considered dynamical system. We have already observed that the use of a multi-arc strategy allows to avoid the computability horizon, therefore in the following we will focus on the uncertainties attainable for the solve-for parameters.

\subsubsection{Pure multi-arc}

Let us first present the results obtained when we apply the pure multi-arc method. Figure 2 shows the cumulative formal uncertainties on the initial conditions of arc $0\left(x_{0}, y_{0}\right)$ and the dynamical parameter $\mu_{0}$ as a function of the total number of arcs $k$ processed, $k=1, \ldots, 2 n+1$, in a log-log plot.

The uncertainty on $\mu_{0}$ decreases as a polynomial function $k^{a}$ of the number of arcs, with $a \sim-0.5$. This is evident if we observe that by definition of pure multi-arc strategy the observed arcs are independent, that is the information on the dynamical parameter contained in each arc is the same in all arcs. As a consequence, as we add more arcs to the fit, the normal matrix $C$ grows as $k$, and the covariance decreases as $k^{-0.5}$, as we found. 


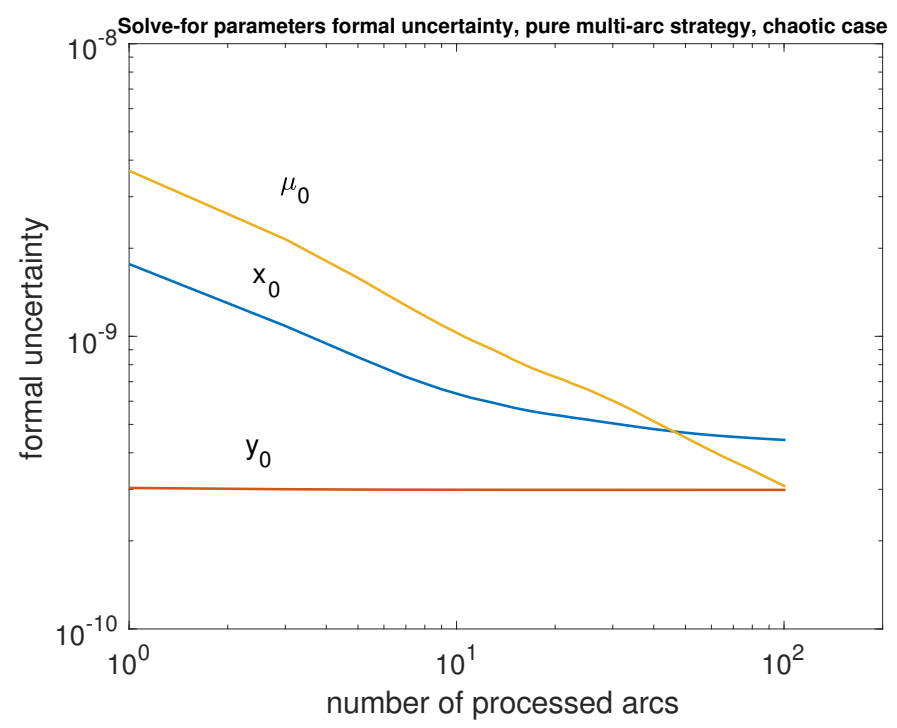

Figure 2: Formal uncertainties of the initial conditions of arc $0\left(x_{0}, y_{0}\right)=(3,0)$ and the dynamical parameter $\mu_{0}=0.5$ as a function of the total number of processed arcs, using a pure multi-arc strategy, chaotic case (log-log plot). The slope of the $\mu_{0}$ formal uncertainty curve is $\sim-0.5$.

The uncertainty on the initial conditions has a different behaviour. Component $x_{0}$ first decreases polynomially (roughly with the same exponent as $\mu_{0}$ ), then it reaches a point where no further improvement is gained, therefore remaining almost constant. Component $y_{0}$ shows no improvement whatsoever. A similar behaviour was expected since by definition in a pure multi-arc strategy the information carried by each observed arc is relevant only for the global parameters and the local parameters of that arc. In particular, it does not affect the local parameters of other arcs. The moderate initial decreasing trend of the $x_{0}$ component is due to correlation with $\mu_{0}$ : improving the knowledge of the latter helps better determine the former. After a number of arcs the correlation is broken and no further improvement for $x_{0}$ is obtainable.

\subsubsection{Constrained multi-arc}

Figure 3 shows the results obtained using the constrained multi-arc method in four different scenarios, corresponding to four different choices of the convergence parameter $\sigma^{*}$, namely $\sigma^{*}=\sigma / 10^{h}$, for $h=1,2,3,4$ respectively. The smaller $\sigma^{*}$, the tighter the constraints between the arcs.

As far as the uncertainties of the initial conditions are concerned, the constrained multi-arc does not change the qualitative behaviour observed in the pure multi-arc case. Both uncertainties decrease at first, then they tend to an asymptotic value. As $\sigma^{*}$ decreases, we observe that the asymptotic value is smaller, thus 
the accuracies attainable for the initial conditions are higher. This is in agreement with how the constrained multi-arc works: smaller values of $\sigma^{*}$ correspond to tighter constraints between subsequent arcs, thus better accuracy in the determination of the orbit. Of course $\sigma^{*}$ cannot be chosen infinitely small, or we would encounter numerical instability. We found indeed that for $\sigma^{*}<\sigma / 10^{4}$ the iterative procedure of differential corrections fails. It is worth remarking that for large values of $\sigma^{*}$ we find similar results to those obtained in the pure multi-arc case, since imposing a loose constraint between two subsequent arcs is in practice equivalent to not constraining them at all.

As regards the dynamical parameter $\mu_{0}$, in all cases its formal uncertainty is a polynomial function of the number of processed arcs, $k^{a}$, where $a<-0.5$. The smaller $\sigma^{*}$ is, the smaller $a$ : in other words, tighter constraints between arcs result in better determination of the dynamical parameter. Note that $a$ is smaller than in the pure multi-arc case for every choice of $\sigma^{*}$, therefore the determination of $\mu_{0}$ benefits from the adoption of a constrained multi-arc technique.

Numerical experiments conducted on other chaotic orbits of the same dynamical system show that such behaviour is in fact general. Although the value $a$ of the slope changes as we change the test orbit, we always find that $a<-0.5$. This result shows that chaotic orbits provide favourable opportunities for estimating free parameters of a dynamical system, and the only way not to deal with numerical computation issues is the use of a constrained multi-arc method.

\subsection{Results: ordered case}

In this section we investigate the results of orbit determination of Chirikov standard map using a multi-arc strategy (pure and constrained) in an ordered case. We chose $\left(x_{0}, y_{0}\right)=(2,2), \mu_{0}=0.5$.

The results obtained using a pure multi-arc strategy are qualitatively similar to the ones obtained with the same strategy in the chaotic case (cf. Fig. 4). The uncertainty in the determination of $\mu_{0}$ decreases indeed as $k^{a}, a \sim-0.5$, where $k$ is the number of arcs, and the initial conditions of arc zero $\left(x_{0}, y_{0}\right)$ do not improve much, for the same reasons explained in Sect. 4.3.1.

Let us go on with the constrained multi-arc strategy. For comparison with the chaotic case, we examined the ordered case similarly to what was done in Sect. 4.3.2: we repeated the same experiment four times, each time with a smaller value of the convergence parameter $\sigma^{*}$. As shown in Fig. 5, in all cases the formal uncertainty of the dynamical parameter $\mu_{0}$ is a polynomial function of the number of $\operatorname{arcs}, k^{a}, a \sim-0.5$. With regard to the initial conditions, their formal uncertainties strongly depend on the choice of $\sigma^{*}$. For larger values of $\sigma^{*}$ they initially tend to decrease, then remain constant, analogously to the chaotic case. The reason is the same as in the previous case: when constraints are rather loose, adding observed arcs to the fit provides little information on other arcs, so the uncertainties 


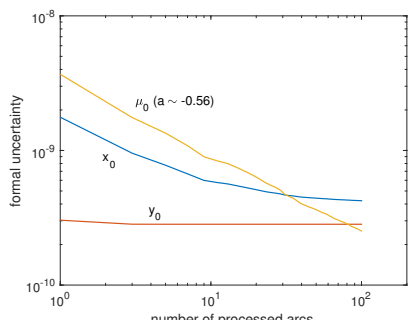

(a) $\sigma^{*}=\sigma / 10$

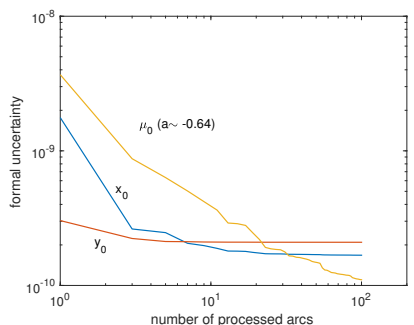

(c) $\sigma^{*}=\sigma / 10^{3}$

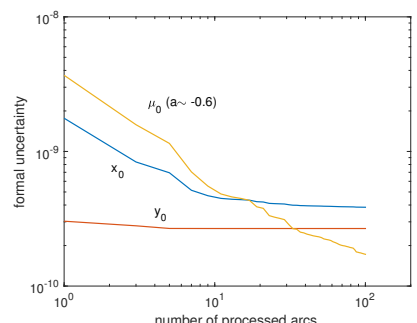

(b) $\sigma^{*}=\sigma / 10^{2}$

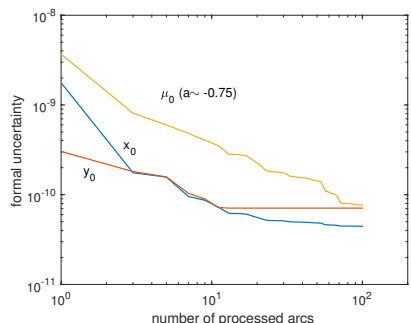

(d) $\sigma^{*}=\sigma / 10^{4}$

Figure 3: Formal uncertainties of the initial conditions of arc $0\left(x_{0}, y_{0}\right)$ and the dynamical parameter $\mu_{0}$ as a function of the total number of processed arcs, using a constrained multi-arc strategy (log-log plot), chaotic case. The four figures refer to four different experiments repeated with the same setting $\left(x_{0}, y_{0}\right)=(3,0), \mu_{0}=0.5$, except for different values of the convergence parameter $\sigma^{*}$ (see formula 7 ). The formal uncertainty of $\mu_{0}$ is a polynomial function of the number of processed arcs, $k^{a}$,. 


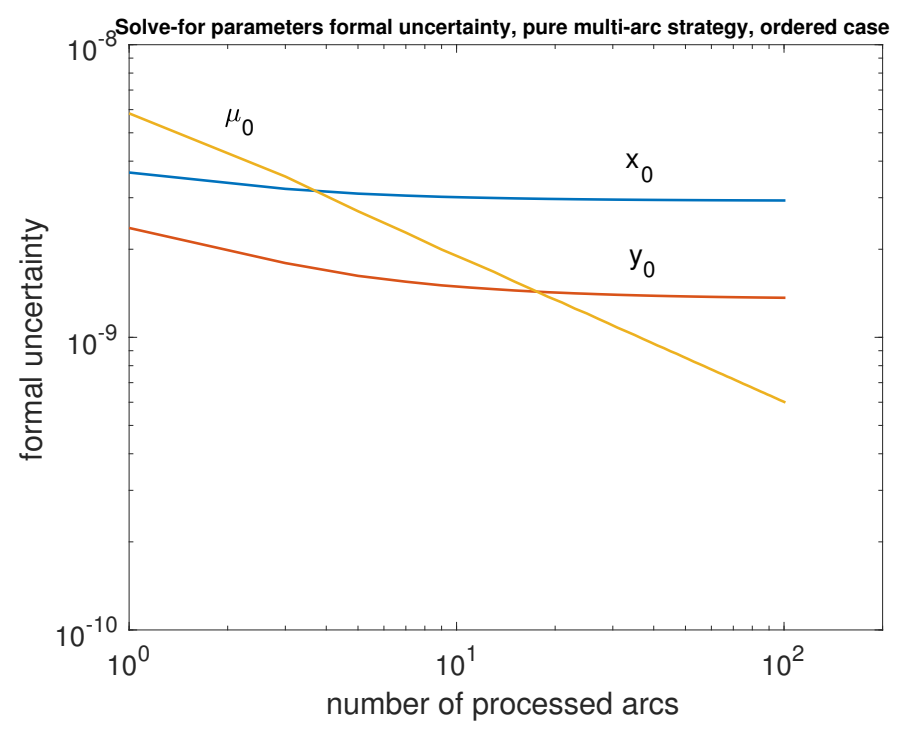

Figure 4: Formal uncertainties of the initial conditions of arc $0\left(x_{0}, y_{0}\right)=(2,2)$ and the dynamical parameter $\mu_{0}=0.5$ as a function of the total number of processed arcs, using a pure multi-arc strategy, ordered case (log-log plot). The slope of the $\mu_{0}$ uncertainty is $\sim-0.5$.

of the initial conditions do not decrease after they reach a certain minimum value. On the other hand, if $\sigma^{*}$ is small enough, the formal uncertainties of the initial condition follow the same law as the dynamical parameter (Fig. 5(d)). This is what happens if we attempt a single-arc solution (cf. Spoto and Milani (2016), Fig. 10), since in the limit for $\sigma^{*} \rightarrow 0$ the constrained multi-arc strategy tends to the single-arc one. Note that in contrast with the chaotic case, in the ordered case no instability is encountered when choosing $\sigma^{*}<\sigma / 10^{4}$. We have studied indeed the case when $\sigma^{*}=\sigma / 10^{5}$, one order of magnitude less than what is possible to do in the chaotic case.

As a final remark, we observe that the determination of $\mu_{0}$ in the ordered case is not affected by the choice of the particular multi-arc strategy used in orbit determination process. As in either case the formal uncertainty of the dynamical parameter is decreasing like $k^{-0.5}$, the constrained multi-arc method does not improve the determination of the parameters when dealing with non-chaotic problems. Nevertheless, it should be adopted in all practical cases when the stability of the computation is compromised by dynamical effects that are difficult to model, for instance in the BepiColombo MORE experiment (Alessi et al. (2012)). 


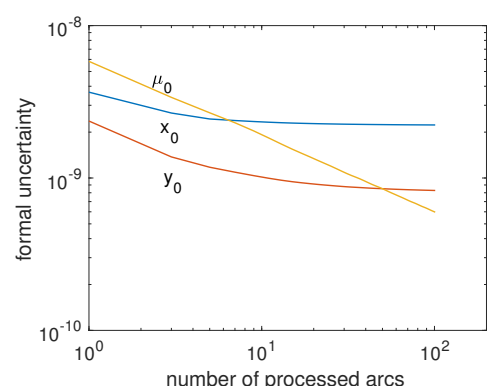

(a) $\sigma^{*}=\sigma / 10$

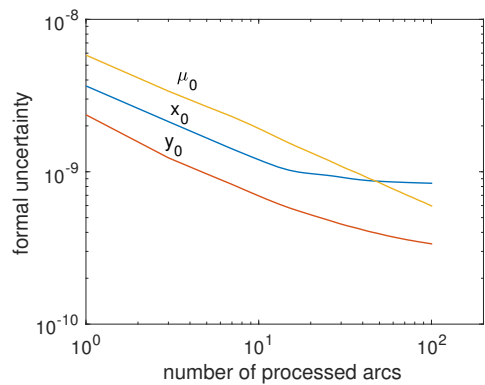

(c) $\sigma^{*}=\sigma / 10^{3}$

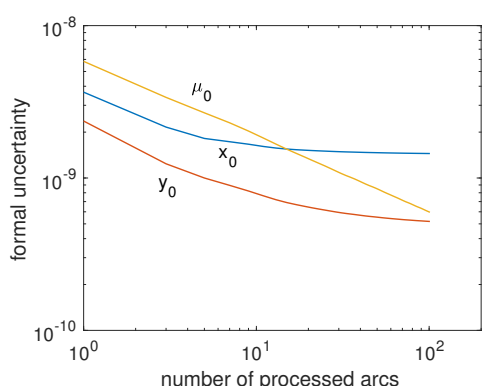

(b) $\sigma^{*}=\sigma / 10^{2}$

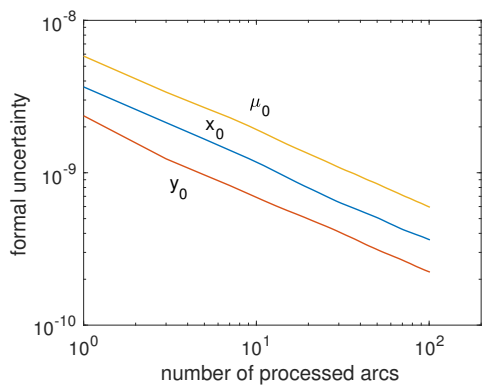

(d) $\sigma^{*}=\sigma / 10^{5}$

Figure 5: Formal uncertainties of the initial conditions of arc $0\left(x_{0}, y_{0}\right)$ and the dynamical parameter $\mu_{0}$ as a function of the total number of processed arcs, using a constrained multi-arc strategy (log-log plot), ordered case. The four figures refer to four different experiments repeated with the same setting, $\left(x_{0}, y_{0}\right)=(2,2), \mu_{0}=0.5$, except for different values of the convergence parameter $\sigma^{*}$ (see formula 7 ). 


\section{Conclusions}

We have applied the classical multi-arc method and the recently-introduced constrained multi-arc method for orbit determination of Chirikov standard map. We simulated observations organized in disjoint arcs and we have solved for a pair of initial conditions for each arc and a global dynamical parameter. We have compared the results obtained with the analysis carried out by Spoto and Milani (2016), where they considered a unique observed arc and solved for a single pair of initial conditions and the same dynamical parameter. Since in the multi-arc strategies the computation of the prediction function is performed arc by arc, by choosing relatively short arcs (about one Lyapunov time of the orbit) we have avoided the computability horizon found in the single-arc strategy when trying to compute a prediction for more than 18 Lyapunov times. We have indeed shown that we are able to compute an orbit of the system for at least 60 Lyapunov times, and the limit is far from being reached. We went even beyond the computability horizon encountered in quadrupole precision, occurring after $\sim 39.2$ Lyapunov times (cf. Spoto and Milani (2016)), which confirms the remarkable strength of this method. Since the uncertainties of the solve-for parameters decrease as the number of observations increases, this allows to achieve higher accuracies in the orbit determination of a chaotic system.

For the determination of a chaotic orbit, in the case of a pure multi-arc strategy, we found that the knowledge of the initial conditions cannot be significantly improved increasing the number of observed arcs. Although we have shown such result for the initial conditions $\left(x_{0}, y_{0}\right)^{0}$ of the central arc, it is also valid for the initial conditions of all the other arcs. On the other hand, the constrained multiarc strategy helps to improve the accuracy on the initial conditions as we add more arcs, provided that we constrain all subsequent arcs to belong to the same trajectory with very small apriori uncertainty $\sigma^{*}$ (as large as a fraction of the observation noise). Moreover, we observed that the smaller $\sigma^{*}$ is, the larger the improvement we obtain. However, we also noted that we are not allowed to choose $\sigma^{*}$ arbitrarily small. This choise would correspond to impose that the arcs are connected with infinite precision; were we allowed to do so, we would also be able to use a single-arc method, as in Spoto and Milani (2016), to process as many observations as desired, which is not the case, as it has already been stated. In fact, there exists a value of $\sigma^{*}$, such that the differential corrections algorithm cannot find a solution. This is the counterpart of the computability horizon for the constrained multi-arc: we can process a number of observations much larger than in the single-arc case, but we are only able to reconstruct a piecewise orbit, whose components are connected within a certain approximation. As regards the determination of the dynamical parameter, we found that its formal uncertainty decreases as a polynomial function of the number of arcs $k$. Such functional law 
is $k^{-0.5}$ when applying a pure multi-arc technique and $k^{a}, a<-0.5$ for the constrained one, $a$ being smaller when tighter constraints between the arcs are applied. This implies that, using the same number of observations, the constrained multiarc method helps to better determine the dynamical parameter with respect to the pure one.

We have also studied an ordered case. We noted that as opposed to the chaotic case we can push orbit determination with constrained multi-arc method to smaller values of the apriori uncertainty $\sigma^{*}$. However, the resulting uncertainty on the dynamical parameter does not depend on the multi-arc technique used: in both cases it decreases as $k^{-0.5}$. On the other hand, we observed that using a constrained multi-arc method improves the determination of the initial conditions of arc 0 , provided that we use a small value of $\sigma^{*}$. For $\sigma^{*}=\sigma / 10^{5}$ such uncertainties behave like $k^{-0.5}$, as in the single arc case.

The product of orbit determination with constrained multi-arc strategy is a collection of orbits, each one relative to an arc, which connect within a certain precision at the central time of the dark period between one arc and the following. One might question the validity of determining such a piecewise orbit in contrast to a single one. Is this "patchwork" of orbits a good approximation of the real one? The answer is positive and comes from an application of the Shadowing Lemma (Anosov (1967), Bowen (1975), Pilyugin (1999) and Spoto and Milani (2016)). Using the standard notation for this theorem, once we fix $\epsilon$, the result of the orbit determination via constrained multi-arc is a $\delta$-psudotrajectory, $\delta=\delta\left(\sigma_{P}^{2}, \sigma, \sigma^{*}\right)>$ 0 and the Shadowing Lemma ensures that there exists an $\epsilon$-shadowing, that is a unique orbit, which approximates the result of the least-squares.

Although we only dealt with a model problem, we believe that the results of this article are of particular relevance in practical applications, especially in the case of space missions exploring the outer planets of the Solar systems and their satellites, like the NASA/ESA/ASI Cassini-Huygens mission to Saturn or the ESA JUICE mission to the jovian system. These kinds of missions are characterized by many close approaches with the planet and its moons ${ }^{5}$, and therefore the orbit of the spacecraft is chaotic (the Lyapunov time being of the same order as the time between two subsequent close encounters). To the best of the authors' knowledge, no single-arc solution has been published yet for any past mission. As already suggested in Dirkx et al. (2017), the use of a constrained multi-arc method would allow to control possible numerical instability arising when the number of observations grows, and at the same time it would ensure significant benefits for the determination of the dynamical parameters, e.g. the spherical harmonics coefficients of the gravity field of the planets/moons, tidal parameters, etc., whose

\footnotetext{
${ }^{5}$ For instance, the Cassini mission had 127 fly-bys with Titan alone.
} 
measurement is the ultimate goal of the Radio Science experiments on board of space missions.

\section{References}

Alessi, E. M., Cicalò, S., Milani, A., Tommei, G., 2012. Desaturation manoeuvres and precise orbit determination for the BepiColombo mission. Monthly Notices of Royal Astronomical Society (423), 2270-2278.

Anosov, D., 1967. Geodesic flows on closed Riemann manifolds with negative curvature. Proceedings of the Steklov Institute of Mathematics 90.

Benkhoff, J., Casteren, J., Hayakawa, H., Fujimoto, M. H., Laakso, H., Novara, M., Ferri, P., Middleton, H., Ziethe, R., 2010. BepiColombo-Comprehensive exploration of Mercury: Mission overview and science goals. Planetary and Space Science 58 (1-2), 2-20.

Bowen, R., 1975. $\omega$-limit sets for axiom A diffeomorphisms. J. Differ. Equ. 18, $333-356$.

Celletti, A., Di Ruzza, S., Lothka, C., Stefanelli, L., 2010. Nearly-integrable dissipative systems and celestial mechanics. The European Physical Journal Special Topics 186 (1), 33-66.

Chirikov, B., 1979. A universal instability of many-dimensional oscillator systems. Phys. Rep. 52, 263.

Chirikov, B., Vecheslavov, V., 1986. Chaotic dynamics of comet Halley. Russian Preprint Inst. Nuclear Physics, 86-184.

Dirkx, D., Gurvits, L., Lainey, V., Lari, G., Milani, A., Cimò, G., BocanegraBahamon, T., Visser, P., 2017. On the contribution of pride-juice to jovian system ephemerides. Planetary and Space Science 147, 14-27.

Lighthill, J., Thompson, J., Sen, A., Last, A., Tritton, D., Mathias, P., 1986. The recently recognized failure of predictability in Newtonian Dynamics [and discussion]. Proceeding of the Royal Society of London, Series A, Mathematical and Physical Sciences 407 (1832), 35-50.

Milani, A., Gronchi, G. F., 2010. Theory of Orbit Determination. Cambridge University Press.

Petrosky, T., 1986. Chaos and cometary clouds in the solar system. Phys. Letters A 117 (328). 
Pilyugin, S. Y., 1999. Shadowing in Dynamical Systems. Lectures Notes in Mathematics 1706.

Schevchenko, I., 2011. The Kepler map in the three-body problem. New Astronomy 16 (2), 94-99.

Spoto, F., Milani, A., 2016. Shadowing Lemma and chaotic orbit determination. Celestial Mechanics and Dynamical Astronomy (124), 295-309. 